\title{
Usefulness of Virtual Expiratory CT Images to Compensate for Respiratory Liver Motion in Ultrasound/CT Image Fusion: A Prospective Study in Patients with Focal Hepatic Lesions
}

\author{
Tae Wook Kang, $M D^{1}$, Min Woo Lee, $M D^{1}$, Dong Ik Cha, MD ${ }^{1}$, Hyun Jung Park, MD², Jun Sung Park, PhD ${ }^{3}$, \\ Won-Chul Bang, PhD 3 , Seon Woo Kim, $\mathrm{PhD}^{4}$ \\ ${ }^{1}$ Department of Radiology and Center for Imaging Science, Samsung Medical Center, Sungkyunkwan University School of Medicine, Seoul, Korea; \\ ${ }^{2}$ Department of Radiology, Chung-Ang University Hospital, Chung-Ang University College of Medicine, Seoul, Korea; ${ }^{3}$ Medical Imaging R\&D Group, \\ Health \& Medical Equipment Business, Samsung Electronics Co., Ltd., Seoul, Korea; ${ }^{4}$ Biostatics and Clinical Epidemiology Center, Samsung Medical \\ Center, Seoul, Korea
}

Objective: To assess whether virtual expiratory (VE)-computed tomography (CT)/ultrasound (US) fusion imaging is more effective than conventional inspiratory (CI)-CT/US fusion imaging for hepatic interventional procedures.

Materials and Methods: This prospective study was approved by the Institutional Review Board, and informed consent was obtained from each patient. In total, 62 patients with focal hepatic lesions referred for hepatic interventional procedures were enrolled. VE-CT images were generated from CI-CT images to reduce the effects of respiration-induced liver motion. The two types of CT images were fused with real-time US images for each patient. The operators scored the visual similarity with the liver anatomy upon initial image fusion and the summative usability of complete image fusion using the respective five-point scales. The time required for complete image fusion and the number of point locks used were also compared.

Results: In comparison with CI-CT/US fusion imaging, VE-CT/US fusion imaging showed significantly higher visual similarity with the liver anatomy on the initial image fusion (mean score, 3.9 vs. $1.7 ; p<0.001$ ) and higher summative usability for complete image fusion (mean score, 4.0 vs. $1.9 ; p<0.001$ ). The required time (mean, 11.1 seconds vs. 22.5 seconds; $p<0.001$ ) and the number of point locks (mean, 1.6 vs. 3.0; $p<0.001$ ) needed for complete image fusion using VE-CT/US fusion imaging were significantly lower than those needed for CI-CT/US fusion imaging.

Conclusion: VE-CT/US fusion imaging is more effective than CI-CT/US fusion imaging for hepatic interventional procedures. Keywords: Ultrasonography; Computed tomography; Fusion imaging; Liver; Respiration

\section{INTRODUCTION}

Fusion imaging fuses pre-acquired three-dimensional (3D) computed tomography (CT)/magnetic resonance imaging and two-dimensional real-time ultrasound (US) images and displays them simultaneously on the US screen (1,
2). Because of these characteristics, fusion imaging has increased the detectability of small focal hepatic lesions (3) and the technical feasibility of percutaneous biopsy or radiofrequency (RF) ablation for hepatic malignancy (4-7). However, despite recent technical advances in fusion imaging, accurate fusion of real-time US and pre-acquired

Received May 16, 2018; accepted after revision September 20, 2018.

Financial (Grant \#PH00132251) and technical support were provided by Samsung Medison and Samsung Electronics (Seoul, Korea).

Corresponding author: Min Woo Lee, MD, Department of Radiology and Center for Imaging Science, Samsung Medical Center,

Sungkyunkwan University School of Medicine, 81 Irwon-ro, Gangnam-gu, Seoul 06351, Korea.

- Tel: (822) 3410-2518 • Fax: (822) 3410-2559• E-mail: leeminwoo0@gmail.com

This is an Open Access article distributed under the terms of the Creative Commons Attribution Non-Commercial License (https:// creativecommons.org/licenses/by-nc/4.0) which permits unrestricted non-commercial use, distribution, and reproduction in any medium, provided the original work is properly cited. 
CT images during interventional procedures poses several technical barriers due to the substantial liver motion and deformation induced by the patients' breathing motion (8). In general, abdominal CT images are obtained during patients' deep inspiration to evaluate the lung base, whereas most US-guided interventional procedures are performed during patients' shallow expiration $(2,9)$. Therefore, real-time US and CT images are obtained in different respiratory phases, making accurate fusion of real-time US and pre-acquired CT images difficult, because the location of a focal hepatic lesion changes with the craniocaudal displacement and intra-organ deformation of the liver induced by a patient's breathing motion (10). This creates a considerable registration error when the current "rigid" registration technique is applied in image fusion because the patients' respiratory phase on the CT images is quite different from that on the US images (11).

To minimize the impact of respiratory liver motion on CT/US fusion imaging, additional CT images during the patient's end-expiratory phase were acquired for use in image fusion in a previous study (12). Indeed, typical inspiratory CT images are not ideal for image fusion with real-time US images. Therefore, herein we describe a method to compensate for respiratory liver motion and deformation by generating virtual expiratory (VE)-CT images from conventional inspiratory (CI)-CT images during the $\mathrm{CT} /$ US image fusion process.

The purpose of this prospective study was to validate the clinical usefulness of this new CT/US image fusion technique for percutaneous biopsy or ablation of focal hepatic lesions by assessment of its summative usability for complete image fusion.

\section{MATERIALS AND METHODS}

\section{Pilot Study}

Using a custom-made simulation software (Blind Test Tool; Samsung Medison, Seoul, Korea), a pilot study was performed using 10 patients to optimize the algorithm for generating VE-CT images that can accurately compensate for respiratory liver motion and thus show better visual similarity with the liver anatomy using VE-CT and US images than using CI-CT and US images. The final version was installed on a commercially available RS80A US system (Samsung Medison) (Fig. 1).
US - 자셕영상 간 유사도 : $\quad$ Very Poor $\bigcirc$ Poor $\bigcirc$ Fair $\bigcirc$ Good $\bigcirc$ Excellent

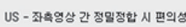
O Very Poor O Poor O Fair O Good O Excellent
US - 우쳥셩상 간 유서도: $\quad O$ Very Poor $\bigcirc$ Poor $O$ Fair $O$ Good $\bigcirc$ Excellent

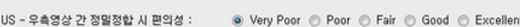

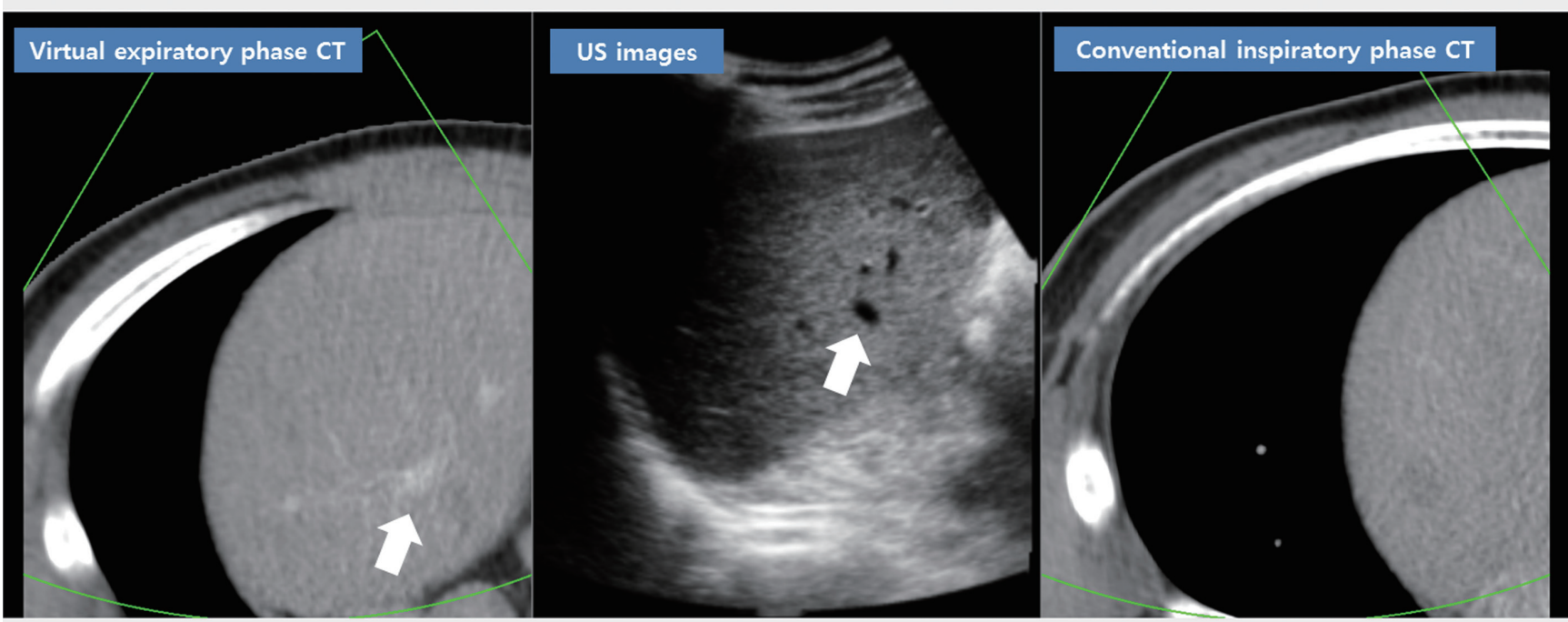

Prev Next o 임정한 0 정일종한

Fig. 1. Pilot study for technical feasibility of VE-CT images using CI-CT images. This is initial alignment of fusion imaging between CT and US images. To easily compare similarity of two images, VE-CT image was positioned on left side of US image and CI-CT image was positioned on right side of US image. Our simulation software (Blind Test Tool; Samsung Medison) shows that hepatic anatomy on US images at rest is more similar to VE-CT than CI-CT upon initial image fusion. Consequently, it is expected that point locks can be performed more easily with VE-CT by selecting right portal vein branch (arrows) for complete image fusion. CI = conventional inspiratory, CT = computed tomography, US = ultrasound, $\mathrm{VE}=$ virtual expiratory 


\section{Study Design}

This was a prospective study performed at a single tertiary academic center (Samsung Medical Center, Sungkyunkwan University, Seoul, Korea). The study protocol was approved by our Institutional Review Board prior to patient enrollment, and all patients gave written informed consent (SMC IRB-2016-04-088). Financial (Grant \#PH00132251) and technical support for this study were provided by Samsung Medison (Seoul, Korea) and Samsung Electronics (Seoul, Korea). However, the authors had complete control of the protocol and the data submitted for publication, which was unbiased by the industries.

\section{Participants}

Between May 2016 and March 2017, patients who met the following inclusion and exclusion criteria and agreed to participate in the current study were prospectively enrolled. The inclusion criteria were as follows: 1 ) patients aged between 20 and 80 years; 2) patients who underwent contrast material-enhanced multiphase CT scans for hepatic focal lesions; 3 ) patients with at least one focal hepatic lesion who were referred to our department for percutaneous biopsy or local ablation therapy, and 4) patients who showed focal hepatic lesions larger than $1 \mathrm{~cm}$ on both US and CT images. The exclusion criteria were 1) a prothrombin time within the abnormal range and platelet count $<50000$ cells $/ \mathrm{mL}^{3}$, indicating a bleeding tendency, and 2) a target lesion that was expected to be located in the blind spots on US on the basis of the location of lung and rib shadows on CT images (13). On the basis of these criteria, 62 consecutive patients ( 35 men, 27 women; mean age, 57.5 years; age range, $25-76$ years) were enrolled (Fig. 2). The baseline characteristics of the 62 patients are summarized in Table 1. Index lesions of fifty patients $(80.6 \%)$ were located in the right hepatic lobe. The most common type of focal hepatic lesions was a malignant tumor (57/62, 91.9\%). Thirteen (20.9\%) patients had liver cirrhosis. Eleven (17.7\%) patients had a previous history of abdominal surgery, including gastrectomy $(n=5)$, hepatic resection $(n=3)$, and pylorus-preserving pancreaticoduodenectomy $(n=3)$.

\section{CT Imaging Acquisition}

CT imaging was performed with one of three multidetector CT machines, including the GE LightSpeed VCT 64 ( $\mathrm{n}$ = 24; GE Healthcare, Waukesha, WI, USA), Philips Brilliance 40 ( $n=21$; Philips Medical Systems, Cleveland, OH, USA), or Aquilion 64 ( $n=10$; Canon Medical Systems, Tokyo, Japan). The scanning parameters were as follows: peak voltage, $120 \mathrm{kVp} ; 189-200 \mathrm{mAs}$, slice thickness, 2.5-3.0 $\mathrm{mm}$; reconstruction interval, $2-3 \mathrm{~mm}$; rotation time, $0.5-$ 0.75 seconds; and pitch, $0.8-1.1$. Hepatic arterial phase

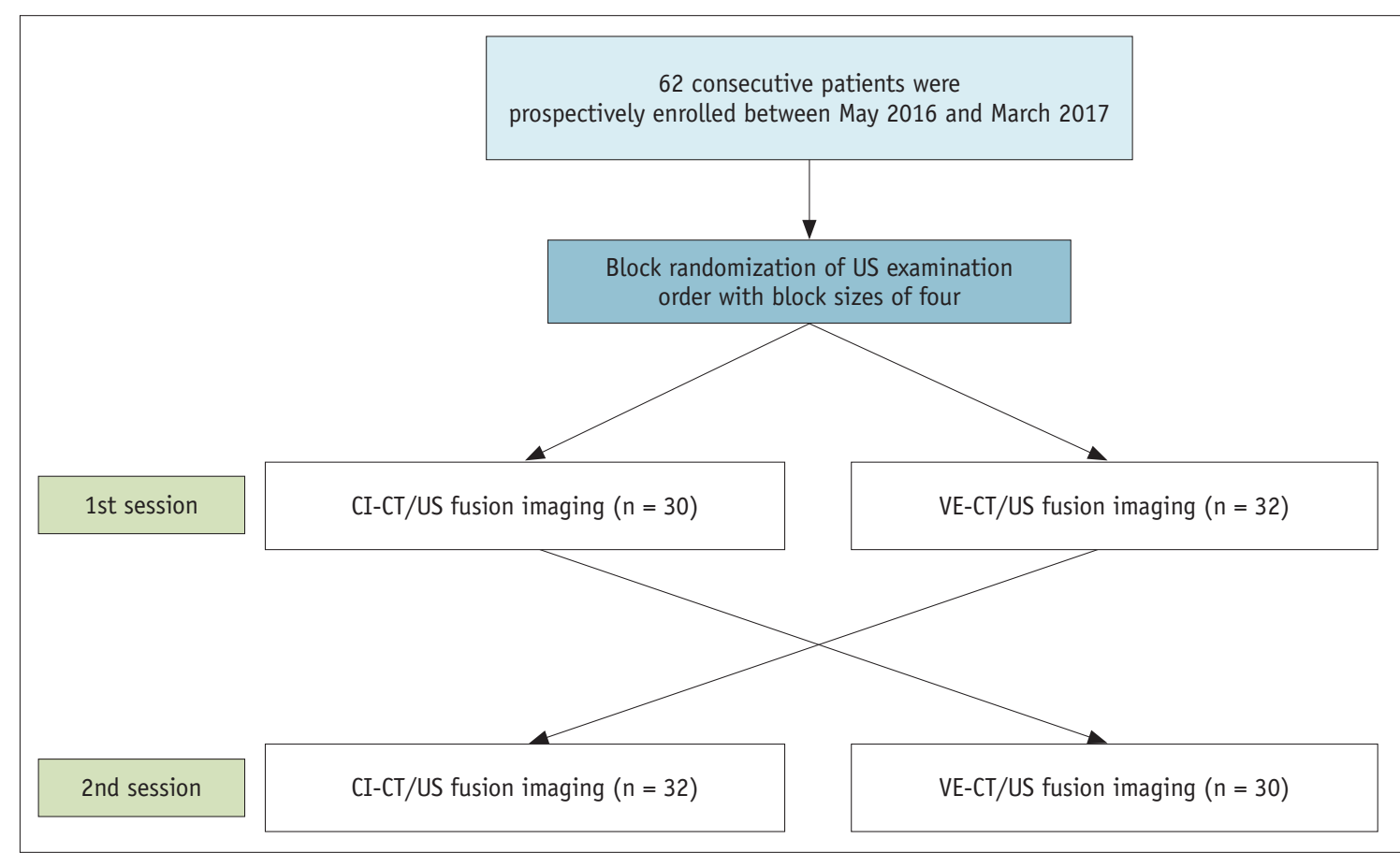

Fig. 2. Study flow diagram. 
Table 1. Baseline Characteristics of 62 Study Participants

\begin{tabular}{|c|c|}
\hline Characteristics & Value \\
\hline Age at enrollment (years)* & $57.5 \pm 11.6(25-76)$ \\
\hline Males & $35(56.5)$ \\
\hline Size of index focal hepatic lesion $(\mathrm{cm})^{*}$ & $3.3 \pm 2.0(0.8-10.0)$ \\
\hline Multiplicity of focal hepatic lesions & $33(53.2)$ \\
\hline \multicolumn{2}{|l|}{$\begin{array}{l}\text { Location of focal hepatic lesions } \\
\text { (Couinaud classification) }\end{array}$} \\
\hline Segment II & $4(6.5)$ \\
\hline Segment III & $7(11.3)$ \\
\hline Segment IV & $1(1.6)$ \\
\hline Segment V & $22(35.4)$ \\
\hline Segment VI & $16(25.8)$ \\
\hline Segment VII & $5(8.1)$ \\
\hline Segment VIII & $7(11.3)$ \\
\hline \multicolumn{2}{|l|}{ Type of focal hepatic lesion } \\
\hline Inflammatory lesion & $3(4.9)$ \\
\hline Benign tumor & $2(3.2)$ \\
\hline Malignant tumor & $57(91.9)$ \\
\hline \multicolumn{2}{|l|}{ Type of hepatic intervention } \\
\hline Liver biopsy & $56(90.3)$ \\
\hline Radiofrequency ablation & $5(8.1)$ \\
\hline Cryoablation & $1(1.6)$ \\
\hline Presence of liver cirrhosis & $13(20.9)$ \\
\hline $\begin{array}{l}\text { Presence of past history of abdominal } \\
\text { surgery }\end{array}$ & $11(17.7)$ \\
\hline
\end{tabular}

Unless indicated otherwise, data are number of patients, with percentages in parentheses. *Data are means and standard deviations with range.

scanning began 20-40 seconds after injection of a contrast agent (Iomeron 300; Bracco, Milan, Italy) with a bolustriggered technique. Portal- and delayed-phase scanning began 70 and 180 seconds after the start of the contrast medium injection, respectively. This was our routine CT protocol for patients with hepatic focal lesions. Seven patients underwent CT examinations at an outside hospital.

\section{US with Fusion Imaging}

The current study was performed with the RS80A US system, which is capable of fusion imaging (S-fusion; Samsung Medison), by two experienced radiologists who had 7 and 5 years of experience, respectively, of fusion imaging for biopsy or ablation treatment at the beginning of this study. S-fusion used two electromagnetic position sensors (3D guidance driveBAY tracker; Ascension Technology Corporation, Shelburne, VT, USA) that were attached to a convex-array transducer and a transmitter. For CT/US fusion imaging, the sweeping auto-registration method was used. The detailed technical specifications of the S-fusion system were identical to those used in previous studies $(14,15)$.

The image fusion process was as follows: 1 ) apply orientation lock; 2) obtain the US volume of the liver dome; and 3) apply additional point lock. The "orientation lock" is a process that automatically recognizes the location of the solar plexus (the junction between the body of the sternum and the xiphoid process) in the CT images and the direction and location of the US transducer relative to the patient. It is performed by placing the transducer on top of the solar plexus in the sagittal plane. Next, the 3D US volume of the liver dome, including the diaphragm, was obtained by "sweeping" the US transducer in the intercostal scan of the right lobe. From the 3D US volume data, the surface of the diaphragm was extracted using the adaptive thresholding technique (16). Meanwhile, the interface between the lung and the adjacent diaphragm was extracted from CT images (17). This registration between US and CT images was automatically achieved by aligning the extracted features from the US and the CT images. Then, real-time US and fused CT images were displayed simultaneously on a splitscreen display (14). Next, a point lock was performed manually for complete image fusion, which is a process of refining the initial registration error. This was done by manually selecting the same point within the same anatomic landmarks (e.g., vessel bifurcation or the focal hepatic lesion) near the target lesion on both real-time US and fused CT images. Finally, the center of the index lesion was marked to complete the process of image fusion (14).

\section{Study Protocol and Generation of VE-CT Images}

After the initial orientation lock, 3D US volume data of the liver were acquired twice, sequentially, at the patient's inspiratory and expiratory phases. This was because two 3D US volume datasets at the two different respiratory phases were required to estimate respiration-induced liver motion and generate a VE-CT image. Liver motion was calculated by aligning the diaphragm from the expiratory and inspiratory US volume data. A CT deformation field was generated by extrapolating the liver motion vectors on the CI-CT image space (Fig. 3). VE-CT images were automatically generated by the US system. Then, two types of image fusion methods were executed sequentially using VE- and CI-CT as reference datasets for each patient in the same session. To minimize the recall bias according to the improvement in awareness with each US exam, the order of fusion methods was randomized. Randomization lists were produced by one author prior to the study. The groups were block-randomized 


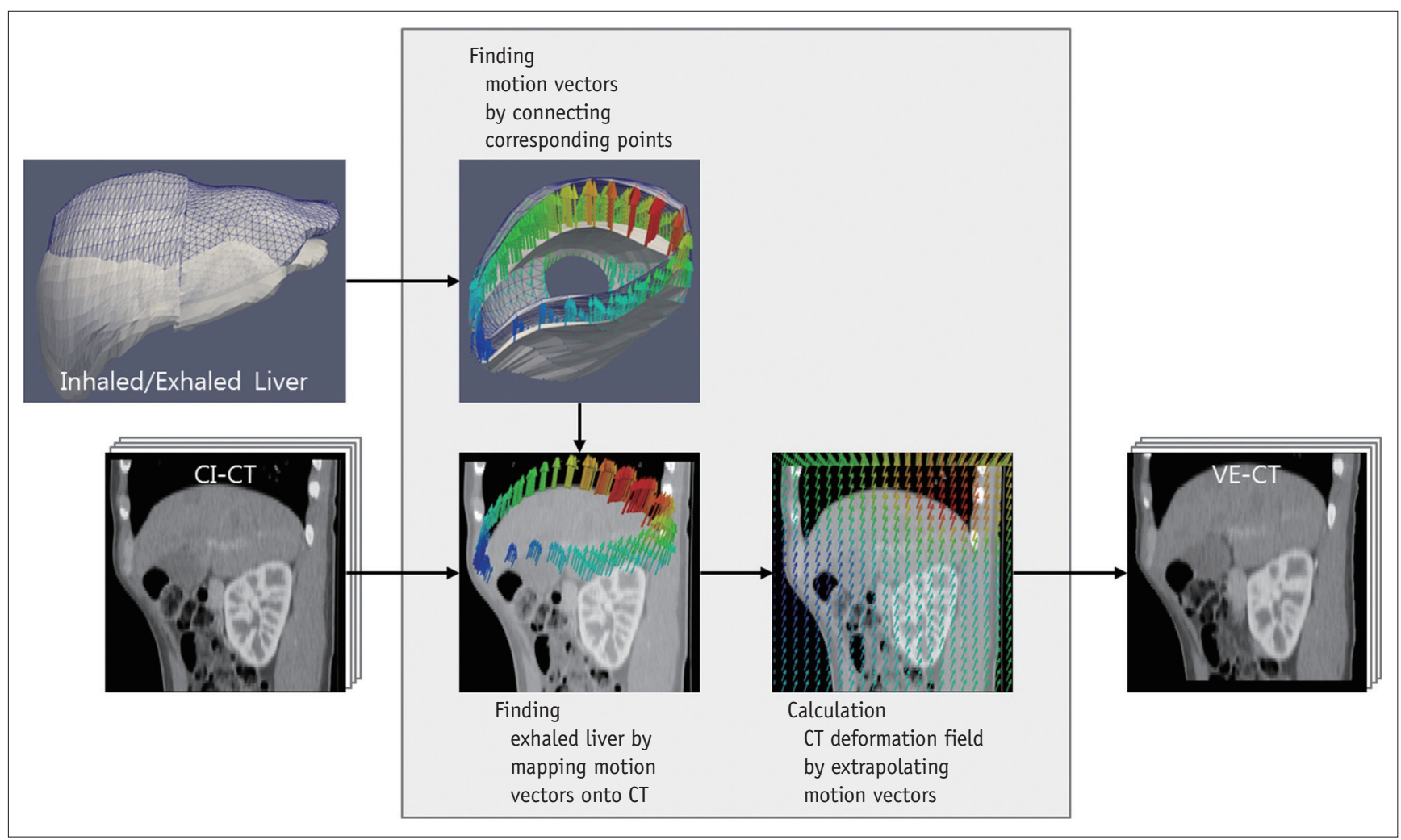

Fig. 3. VE-CT generation using CI-CT and estimated liver motion.

Table 2. Definitions of Outcomes in Study

\begin{tabular}{|c|c|c|c|}
\hline Process & Rating Categories & Descriptions & Rating (1-5) \\
\hline Initial image fusion & Visual similarity & $\begin{array}{l}\text { To evaluate visual similarity of diaphragm, liver contour, } \\
\text { axis angle, intrahepatic vessels, and target lesion upon } \\
\text { initial image fusion between real-time US and CT images }\end{array}$ & $\begin{array}{l}\text { 1. Highly dissimilar } \\
\text { 2. Dissimilar } \\
\text { 3. Average similar } \\
\text { 4. Similar } \\
\text { 5. Highly similar }\end{array}$ \\
\hline Complete image fusion & Summative usability & $\begin{array}{l}\text { To assess summative usability for fine registration using } \\
\text { anatomic landmarks and target lesion }\end{array}$ & $\begin{array}{l}\text { 1. Really not confident } \\
\text { 2. Not confident } \\
\text { 3. Not sure } \\
\text { 4. Confident } \\
\text { 5. Really confident }\end{array}$ \\
\hline
\end{tabular}

Real-time US images at time of end-expiratory phase were used as reference standards for comparison between CT and US. CT = computed tomography, US = ultrasound

with block sizes of four. Randomization was performed on the examination day.

The operator selected one of the reference datasets (either CI-CT or VE-CT images) and compared the shape of the liver, the locations of the landmark vessels, and the target lesion between the real-time US and the fused CT images. The operators scored the similarity of the initially fused images using a five-point scale for both VE-CT/US and CI-CT/US (Table 2). Then, additional point locks were performed to refine the initial registration with each CT dataset at the end-expiratory phase because interventional procedures are usually performed at this respiratory phase. During additional point locks, the summative usability of complete image fusion was also assessed with a five-point scale (Table 2). Portal phase CT images, when hepatic vessels were clearly identified, were used as the reference datasets if possible. However, if the index tumor was not clearly visible at this phase, arterial or 3-minute delayed-phase CT images were used instead (Fig. 4). 


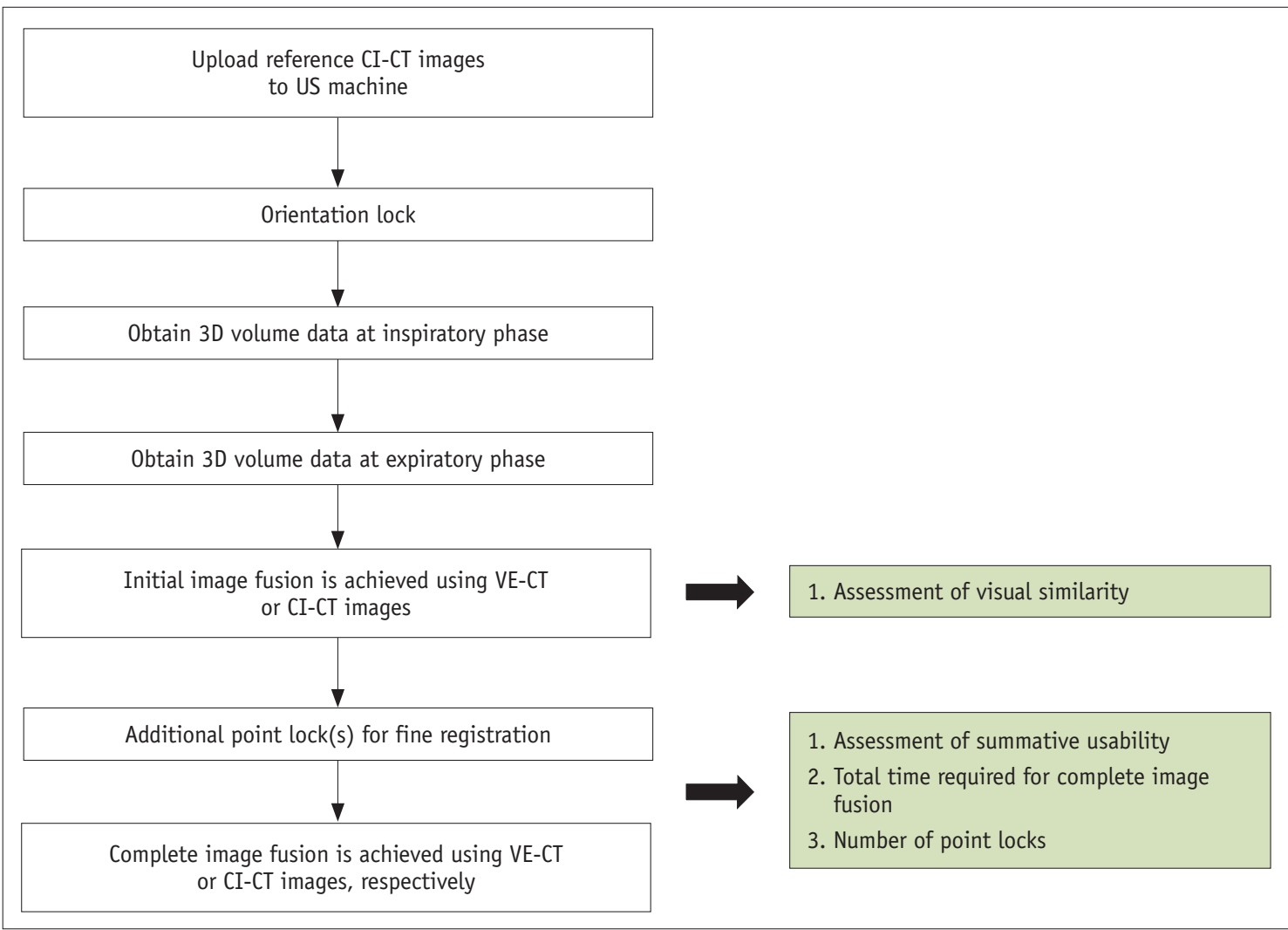

Fig. 4. Process of image fusion and outcome evaluation. 3D = three-dimensional

\section{Outcomes}

Our primary outcome was the summative usability of complete image fusion. The secondary outcomes were the visual similarity of the liver anatomy between real-time US and initially fused CT images, the number of additional point locks required for complete image fusion, and the time required for complete image fusion. If a patient had multiple focal hepatic lesions, the largest one was selected as an index lesion for evaluation of summative usability. The time required for complete image fusion was defined as the total elapsed time from the display of real-time US and initially fused CT images to the endpoint of the last point lock. The number of additional point locks and the time required for complete image fusion was automatically calculated using in-house software after each exam (Elapse Time Calculation Tool; Version 1.0, Samsung Medison) (14). In this study, initial image fusion refers to image fusion achieved by applying the sweeping auto-registration method before applying any point lock. Complete image fusion refers to image fusion after performing point locks as much as needed to refine the registration error.

\section{Sample Size Calculation}

The primary hypothesis was that the proportions of scores of 4 or 5 for summative usability in complete image fusion between VE-CT and CI-CT images would be different. Based on our pilot study, the proportion of scores of 4 or 5 was $40 \%$ using CI-CT images and was expected to be at least $60 \%$ using VE-CT images. The proportions of superior performance (scores of either 4 or 5 ) were assumed to be $5 \%$ and $25 \%$ in the CI-CT and VE-CT images, respectively, and the variance of the proportion difference between the two methods was estimated to be 0.50 . According to the information given, the sample size needed in order to achieve $80 \%$ power under a significance level of $5 \%$ was 50 subjects. Assuming a dropout rate of $20 \%, 62$ subjects would have to be enrolled.

\section{Statistical Analysis}

All clinical data are described using means with standard deviation, medians with range, or frequencies with proportions. Because this was a crossover prospective study using VE-CT and CI-CT images, generalized estimating equations were used for categorical variables and a mixed linear model was used for continuous variables. In terms of 
VE-CT/US image fusion, a subgroup analysis of outcomes was performed according to the location of the target lesion (right vs. left hepatic lobe), the presence of liver cirrhosis, and the presence of a history of abdominal surgery because these factors could affect the degree of intra-organ deformation or liver displacement $(8,18)$. A $p$ value $<$

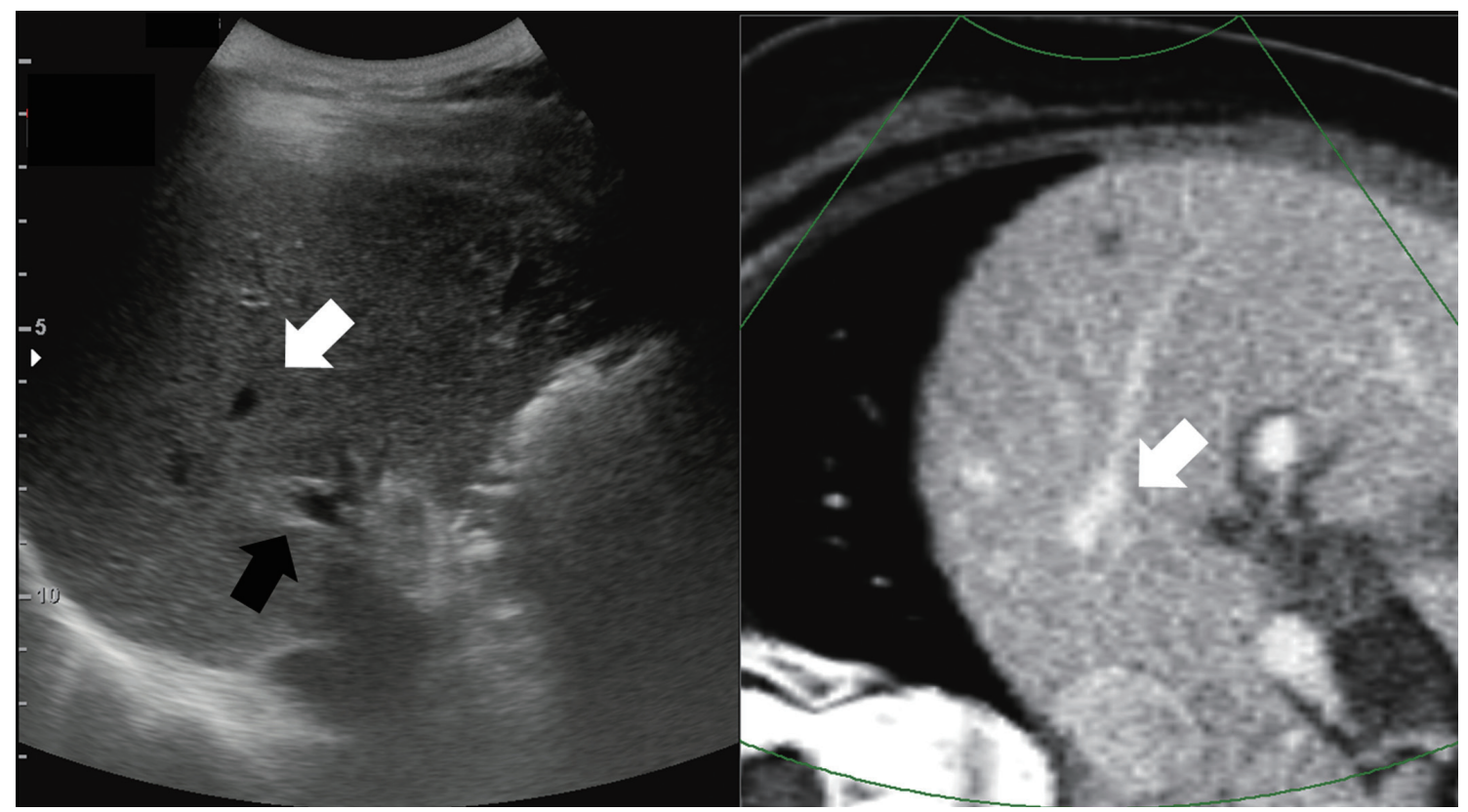

A

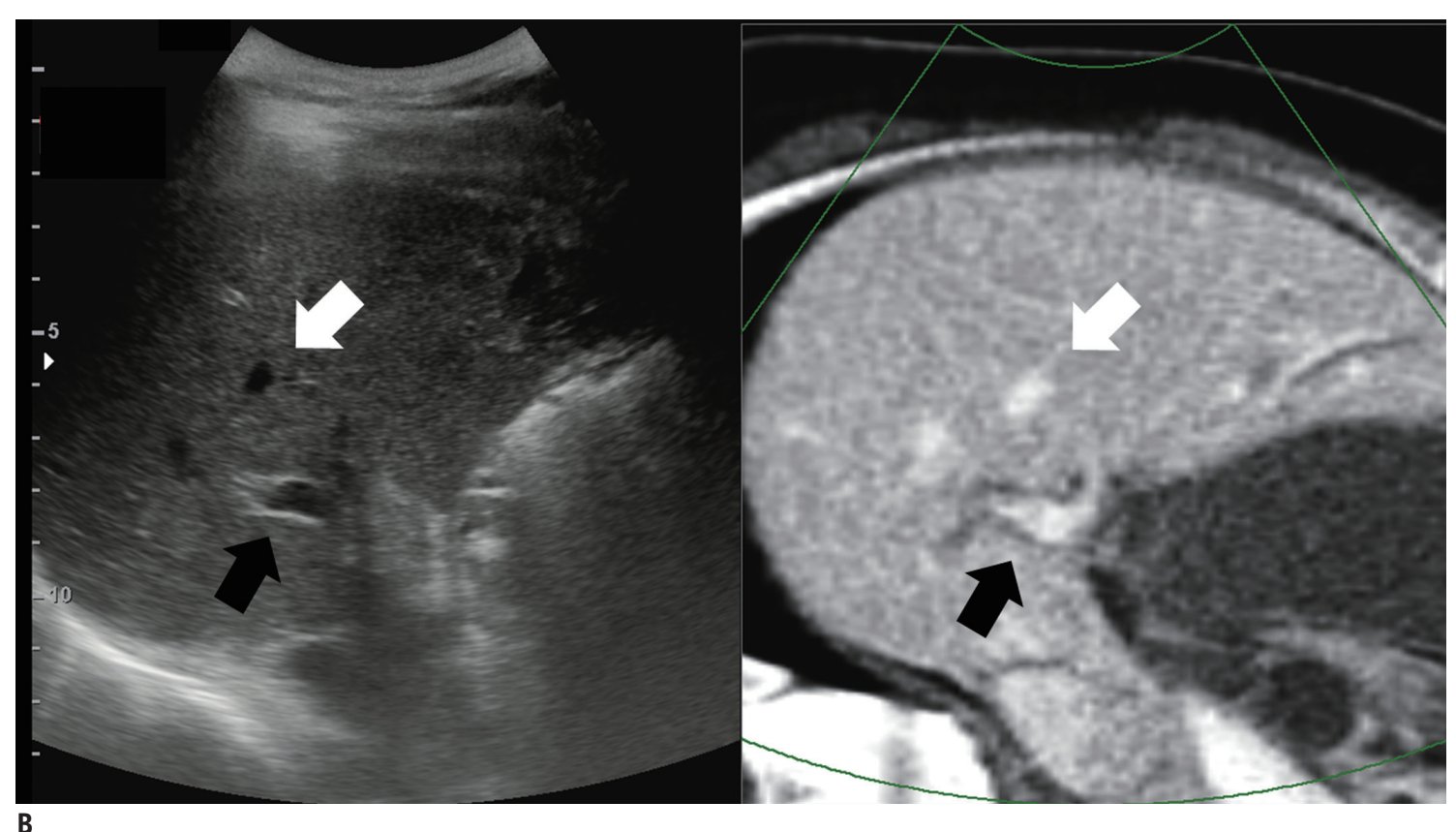

Fig. 5. Improved visual similarity after initial image fusion in VE-CT/US fusion imaging.

A. 54-year-old-woman with hepatic metastasis from pancreatic cancer. On CI-CT/US fusion imaging before applying any point lock, anatomical relationship of right hepatic vein (white arrows) and right portal vein (black arrow) is quite different between real-time US images and CI-CT images. In CI-CT image, right portal vein is not visible due to misregistration. Total time elapsed for complete image fusion was 27 seconds. Scores for visual similarity and summative usability were 2 and 3, respectively. B. When using VE-CT images as reference dataset, hepatic vascular anatomy on real-time US image was quite similar to that on VE-CT image due to compensation of respiration-induced liver motion. Consequently, total time required for complete image fusion was only 6 seconds. In this case, VE-CT/US image fusion was performed first according to results of randomization. Scores for visual similarity and summative usability were 5 and 5 , respectively. Right hepatic vein (white arrows) and right portal vein (black arrows). 
0.05 was considered to indicate statistical significance. All analyses were performed using a statistical package (SPSS, version 17.0 for Windows, SPSS Inc., Chicago, IL, USA).

\section{RESULTS}

Visual Similarity with the Liver Anatomy and Summative Usability According to the Fusion Methods

VE-CT images were generated in all subjects without any

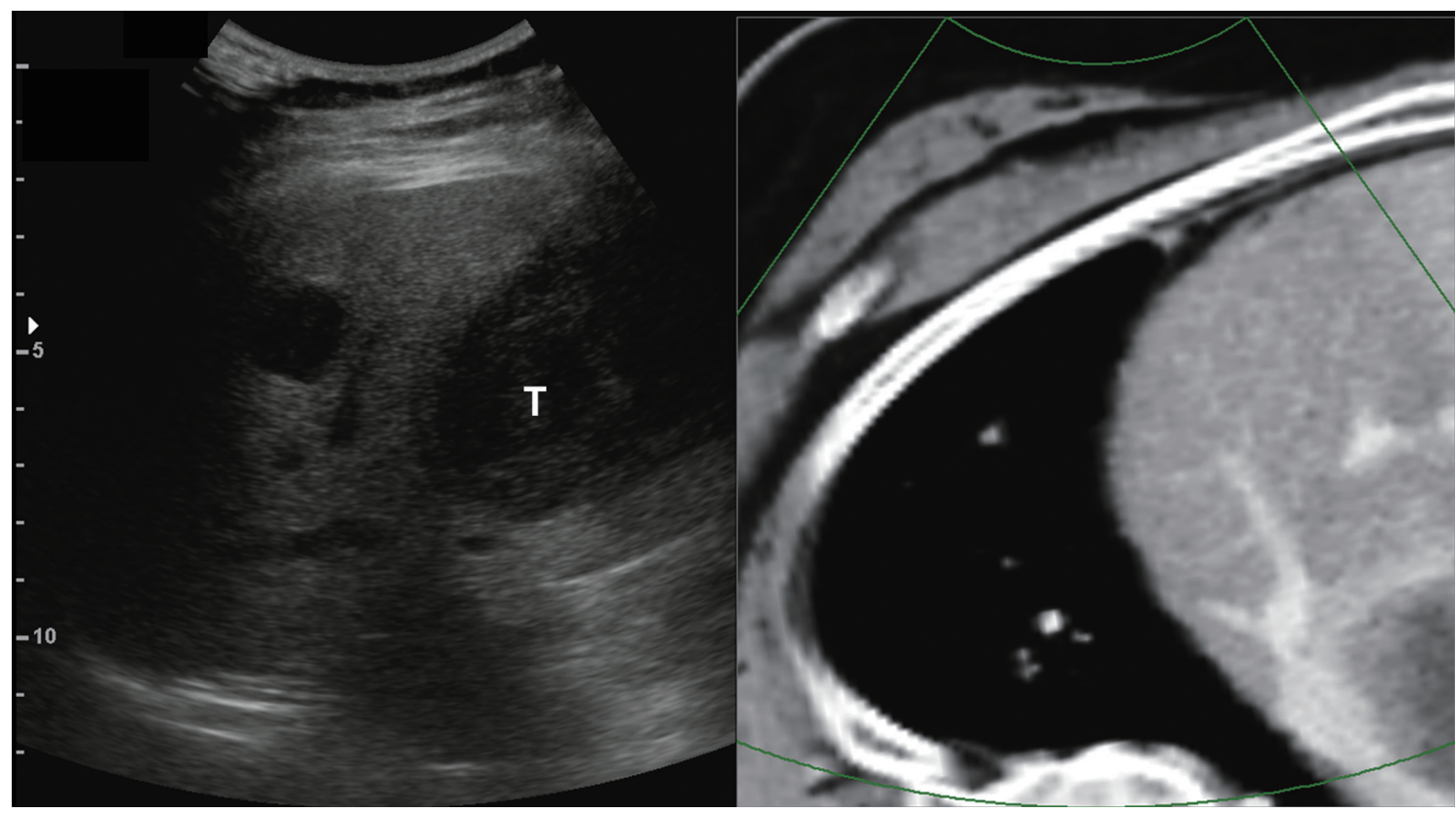

A

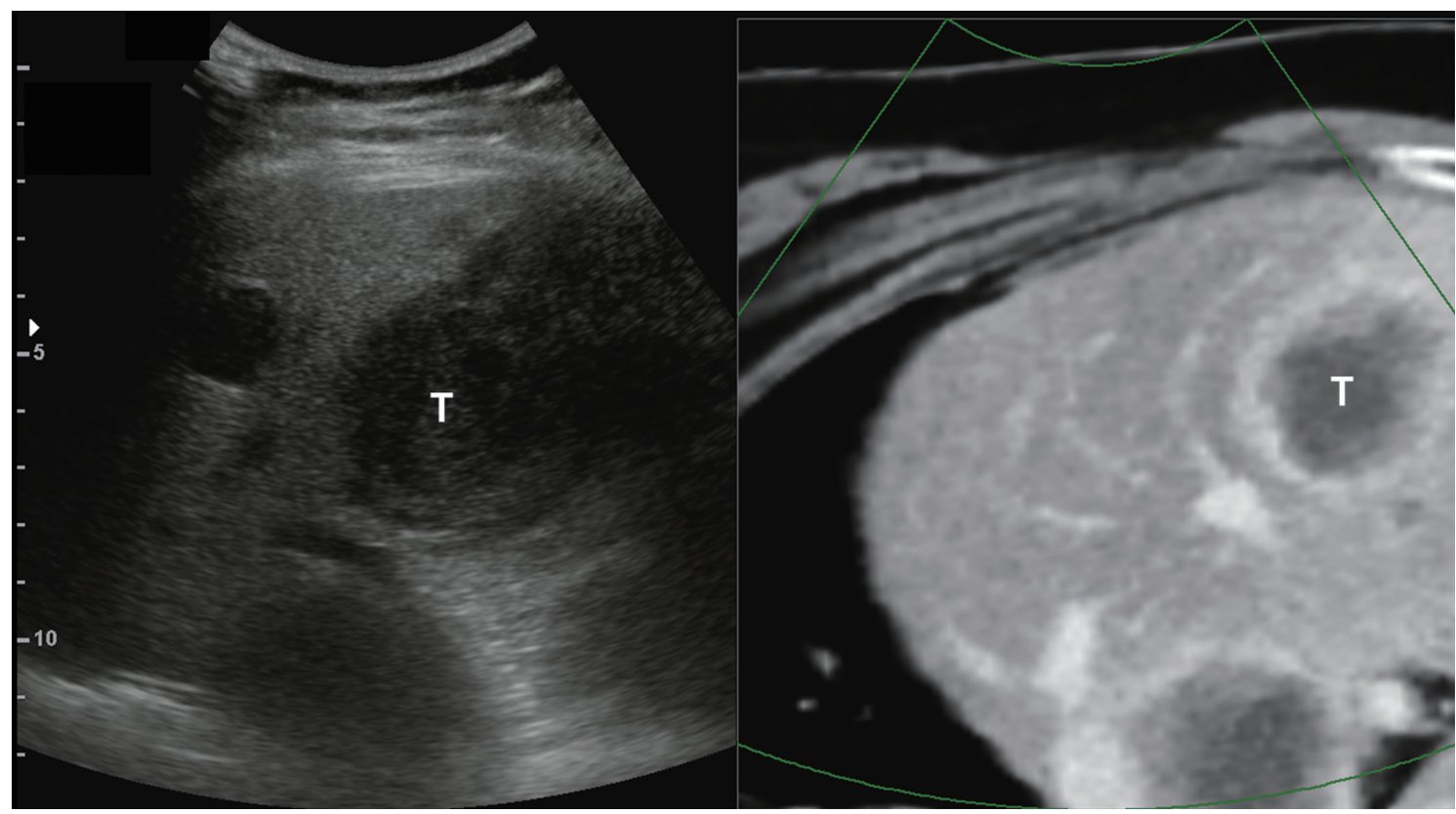

Fig. 6. Improved summative usability for complete image fusion in VE-CT/US fusion imaging.

A. 50-year-old-woman with multiple hepatic metastases from colon cancer. On CI-CT/US fusion imaging before applying any point lock, index T was invisible on CI-CT image upon initial registration. Therefore, two additional point locks were needed for complete image fusion. Total time required for complete image fusion was 10 seconds. Scores for visual similarity and summative usability were 2 and 2, respectively. B. On VE-CT/ US fusion imaging before applying any point lock, index T was clearly visible at corresponding site and only one point lock was enough for fine registration. Total time required for complete image fusion was 4 seconds. In this case, VE-CT/US image fusion was performed first according to results of randomization. Scores for visual similarity and summative usability were 4 and 5 , respectively. $T=$ tumor 
technical failures. The mean time needed for generating VE-CT images with the US system was 36.1 seconds (range, 19-55 seconds). The visual similarity of the initial CT/US fusion imaging was better in the VE-CT/US images than in the CI-CT/US images (mean score, 3.9; range, 3-5 vs. mean score, 1.7 ; range, $1-4 ; p<0.001)$. The summative usability of complete image fusion was also better in the VE-CT/US images than in the CI-CT/US images (mean score, 4.0; range, $2-5$ vs. mean score, 1.9 ; range, $1-4 ; p<0.001$ ) (Figs. 5, 6).

\section{The Time and Number of Point Locks Required for} Complete Image Fusion

After automatic initial image fusion, the time required for complete image fusion by applying point locks was significantly shorter with the VE-CT/US images than with the CI-CT/US images (mean, 11.1 seconds; range, 5-27 seconds vs. mean, 22.5 seconds; range, 10-72 seconds; $p$ $<0.001$ ). In addition, the number of point locks needed for complete image fusion was significantly smaller using the VE-CT/US images than that using the CI-CT/US images (mean, 1.6; range, $1-3$ vs. mean, 3.0; range, $1-6 ; p<0.001$ ).

\section{Subgroup Analysis of Outcomes According to the}

Location, Presence of Liver Cirrhosis, and History of

\section{Abdominal Surgery}

In terms of VE-CT/US image fusion, both visual similarity with the initial image fusion and summative usability of complete image fusion were not significantly different in all subgroup analyses based on tumor location, presence of liver cirrhosis, and previous history of abdominal surgery (all $p$ values $>0.05$ ) (Table 3 ).

\section{DISCUSSION}

Liver motion and deformation as a result of respiratory movement are important issues in tracking liver tumors, not only for US-guided interventional procedures, but also for other treatment options such as external radiation therapy $(3,8,19)$. Fusion of two imaging modalities with different respiratory phases, such as CT/US image fusion, using rigid registration can result in considerable registration errors (11). The commercially available fusion imaging systems to date use real-time US and pre-acquired CT/magnetic resonance images and are based on rigid registration, which incompletely compensates for patient respiration and movement $(17,20)$. For this reason, generation of VE-CT images from CI-CT data can be useful for image fusion. Our study showed that VE-CT/US fusion imaging provided better similarity with the liver anatomy upon initial image fusion and better summative usability of complete image fusion in comparison with CI-CT/US fusion imaging.

Even if the liver moves as a rigid body without internal deformation, craniocaudal movement of the liver can be up to $2.5 \mathrm{~cm}$ during a normal respiration cycle (21). Therefore, registration errors in fusion imaging between pre-acquired CT images and real-time US images is inevitable. In line with this assumption, a previous study showed that the registration error and motion of focal hepatic lesions during fusion of real-time US with CT images were affected by the degree of respiratory movement and the location of an index lesion (11). In addition, subcapsular location of a tumor was a significant factor responsible for mistargeting even after fusion imaging-guided RF ablation because liver deformation and displacement by the patient's breathing motion were more apparent in the peripheral liver than in the central liver $(8,22)$.

In our study, VE-CT images could be generated effectively from CI-CT images by motion vector analysis of CT deformation. This was helpful to overcome the respiratory phase issue for CT/US fusion imaging because VE-CT

Table 3. Subgroup Analysis of Outcomes According to Location, Liver Cirrhosis, and Past History of Abdominal Surgery in VE-CT/ US Image Fusion

\begin{tabular}{|c|c|c|c|c|}
\hline & Visual Similarity & $P$ & Summative Usability & $P$ \\
\hline Location & & 0.816 & & 0.951 \\
\hline Right hepatic lobe & $3.9 \pm 0.9(2-5)$ & & $4.1 \pm 0.8(2-5)$ & \\
\hline Left hepatic lobe & $3.6 \pm 0.8(3-5)$ & & $3.8 \pm 0.7(3-5)$ & \\
\hline Hepatic fibrosis & & 0.607 & & 0.209 \\
\hline Cirrhosis & $3.8 \pm 1.0(2-5)$ & & $4.1 \pm 0.9(3-5)$ & \\
\hline Non-cirrhosis & $3.9 \pm 0.8(2-5)$ & & $4.0 \pm 0.7(2-5)$ & \\
\hline Abdominal adhesion & & 0.384 & & 0.833 \\
\hline Abdominal surgery group & $4.0 \pm 0.9(3-5)$ & & $4.1 \pm 0.8(3-5)$ & \\
\hline Non-surgery group & $3.9 \pm 0.9(2-5)$ & & $4.0 \pm 0.8(2-5)$ & \\
\hline
\end{tabular}

Data are means and standard deviations with range in parentheses. $V E=$ virtual expiratory 
images were based on patients' expiratory phase, which is quite similar to the respiratory phase during the actual interventional procedures. Therefore, the discrepancy in respiration-related liver motion between real-time US and VE-CT would be much smaller than that between US and CI-CT. As expected, the summative usability of complete $\mathrm{CT} / \mathrm{US}$ image fusion was much higher using VE-CT images than that using CI-CT images. The advantages of VE-CT images as a reference dataset include reduction of the time needed for fine registration and the smaller number of manual point locks needed for complete image fusion due to the improvement in visual similarity of the liver anatomy between real-time US and fused CT images. However, as this study was performed by experienced radiologists and since the time needed for complete image fusion depends on the operator's experience, the usefulness of VE-CT as a reference dataset for image fusion warrants further validation with less-experienced operators.

Before conducting the current investigation, we hypothesized that there may be a difference in visual similarity according to the tumor location because cardiac motion was not taken into consideration in our algorithm for generating VE-CT images. However, our subgroup analysis showed that the tumor location (right vs. left hepatic lobe) did not affect the visual similarity with the liver anatomy upon initial image fusion. This may be explained by the fact that cardiac-induced liver motion mainly occurs in the limited area underneath the heart (23). Interestingly, the presence of liver cirrhosis (8) and past history of abdominal surgery $(18,24)$, both of which are associated with greater rigidity and less movement of the liver, also did not affect the visual similarity and summative usability when realtime US images were fused with VE-CT images in this study. Although the reasons for this are not clearly understood, our algorithm for generation of VE-CT images based on motion vector analysis of CT deformation using the diaphragm movement from the expiratory and inspiratory US volume data seems to effectively work in various situations including liver deformation or limited liver movement.

This study has several limitations. First, although we defined the outcomes in detail prior to the current study, visual similarity and summative usability were subjectively rated by each operator and interobserver agreement was not assessed. This was inevitable as the process of image fusion was performed by each operator and the time taken for the entire process was not short enough to be stored as cine files. Second, due to the prospective crossover study design, we did not evaluate clinical outcomes such as the technical success rate of liver biopsy or the local tumor progression rate after RF ablation according to the use of VE-CT. Third, quantitative measurement of registration errors after complete image fusion was not performed because registration errors can be reduced by applying point locks at the center of the tumor regardless of the reference dataset. Even though CI-CT could be used as a reference dataset, the patients cannot hold their breaths at deep inspiration long enough for the operator to position an applicator into the tumor. In addition, rigid registration with CI-CT may be rather time-consuming as the liver anatomy is not identical between real-time US and pre-acquired CI-CT. In this context, VE-CT would be an alternative reference dataset for image fusion as it can be easily generated by sweeping the liver with a US transducer. Although VE-CT does not provide non-rigid registration reflecting real-time anatomic changes of the liver, we think that our VE-CT technique will be a cornerstone for developing real-time non-rigid registration of pre-acquired CT and real-time US images. We hope that non-rigid registration is available in the near future.

In summary, VE-CT/US images provide better similarity with the liver anatomy upon initial image fusion compared with CI-CT/US images. This can lead to an increase in the summative usability of complete image fusion with a shorter registration time and a smaller number of point locks for hepatic interventional procedures.

\section{Conflicts of Interest}

Jun Sung Park and Won-Chul Bang received support in the form of salaries from Samsung Electronics. Min Woo Lee is a consultant for Samsung Medison. All other authors declare that they have no conflicts of interest.

\author{
ORCID \\ Min Woo Lee \\ https://orcid.org/0000-0001-9048-9011 \\ Tae Wook Kang \\ https://orcid.org/0000-0002-0725-8317
}

\section{REFERENCES}

1. Yang M, Ding H, Kang J, Zhu L, Wang G. Subject-specific real-time respiratory liver motion compensation method for ultrasound-MRI/CT fusion imaging. Int $\mathrm{J}$ Comput Assist Radiol Surg 2015;10:517-529

2. Lee MW. Fusion imaging of real-time ultrasonography with CT 
or MRI for hepatic intervention. Ultrasonography 2014;33:227239

3. Park HJ, Lee MW, Lee MH, Hwang J, Kang TW, Lim S, et al. Fusion imaging-guided percutaneous biopsy of focal hepatic lesions with poor conspicuity on conventional sonography. J Ultrasound Med 2013;32:1557-1564

4. Ahn SJ, Lee JM, Lee DH, Lee SM, Yoon JH, Kim YJ, et al. Real-time US-CT/MR fusion imaging for percutaneous radiofrequency ablation of hepatocellular carcinoma. $\mathrm{J}$ Hepatol 2017;66:347-354

5. Jung EM, Friedrich C, Hoffstetter P, Dendl LM, Klebl F, Agha A, et al. Volume navigation with contrast enhanced ultrasound and image fusion for percutaneous interventions: first results. PLoS One 2012; 7:e33956

6. Lee DH, Lee JM. Recent advances in the image-guided tumor ablation of liver malignancies: radiofrequency ablation with multiple electrodes, real-time multimodality fusion imaging, and new energy sources. Korean J Radiol 2018;19:545-559

7. Kim JW, Shin SS, Heo SH, Hong JH, Lim HS, Seon HJ, et al. Ultrasound-guided percutaneous radiofrequency ablation of liver tumors: how we do it safely and completely. Korean J Radiol 2015;16:1226-1239

8. Paulsson AK, Yom SS, Anwar M, Pinnaduwage D, Sudhyadhom A, Gottschalk AR, et al. Respiration-induced intraorgan deformation of the liver. Technol Cancer Res Treat 2017:1533034616687193

9. Cha DI, Lee MW, Kang TW, Oh YT, Jeong JY, Chang JW, et al. Comparison between CT and MR images as more favorable reference data sets for fusion imaging-guided radiofrequency ablation or biopsy of hepatic lesions: a prospective study with focus on patient's respiration. Cardiovasc Intervent Radiol 2017;40:1567-1575

10. Rohlfing T, Maurer CR Jr, O'Dell WG, Zhong J. Modeling liver motion and deformation during the respiratory cycle using intensity-based nonrigid registration of gated MR images. Med Phys 2004;31:427-432

11. Lee MW, Park HJ, Kang TW, Ryu J, Bang WC, Lee B, et al. Image fusion of real-time ultrasonography with computed tomography: factors affecting the registration error and motion of focal hepatic lesions. Ultrasound Med Biol 2017;43:2024-2032

12. Mauri G, Cova L, De Beni S, Ierace T, Tondolo T, Cerri A, et al. Real-time US-CT/MRI image fusion for guidance of thermal ablation of liver tumors undetectable with US: results in 295 cases. Cardiovasc Intervent Radiol 2015;38:143-151

13. Kang TW, Rhim H, Lee MW, Kim W, Park JG. Predicting coverage of transverse subcostal sonography with the use of previous computed tomography before a sonographic liver examination: a prospective study. J Ultrasound Med 2013;32:2053-2061

14. Cha DI, Lee MW, Kim AY, Kang TW, Oh YT, Jeong JY, et al. Automatic image fusion of real-time ultrasound with computed tomography images: a prospective comparison between two auto-registration methods. Acta Radiol 2017;58:1349-1357

15. Cha DI, Lee MW, Song KD, Oh YT, Jeong JY, Chang JW, et al. A prospective comparison between auto-registration and manual registration of real-time ultrasound with MR images for percutaneous ablation or biopsy of hepatic lesions. Abdom Radiol (NY) 2017;42:1799-1808

16. Bobin J, Starck JL, Fadili JM, Moudden Y, Donoho DL. Morphological component analysis: an adaptive thresholding strategy. IEEE Trans Image Process 2007;16:2675-2681

17. Che C, Mathai TS, Galeotti J. Ultrasound registration: a review. Methods 2017;115:128-143

18. Kang TW, Lee MW, Hye MJ, Song KD, Lim S, Rhim H, et al. Percutaneous radiofrequency ablation of hepatic tumours: factors affecting technical failure of artificial ascites formation using an angiosheath. Clin Radiol 2014;69:12491258

19. Yu JI, Kim JS, Park HC, Lim DH, Han YY, Lim HC, et al. Evaluation of anatomical landmark position differences between respiration-gated MRI and four-dimensional CT for radiation therapy in patients with hepatocellular carcinoma. Br J Radiol 2013;86:20120221

20. Ewertsen C, Săftoiu A, Gruionu LG, Karstrup S, Nielsen MB. Real-time image fusion involving diagnostic ultrasound. $A J R$ Am J Roentgenol 2013;200:W249-W255

21. Suramo I, Päivänsalo M, Myllylä V. Cranio-caudal movements of the liver, pancreas and kidneys in respiration. Acta Radiol Diagn (Stockh) 1984;25:129-131

22. Lim S, Lee MW, Rhim H, Cha DI, Kang TW, Min JH, et al. Mistargeting after fusion imaging-guided percutaneous radiofrequency ablation of hepatocellular carcinomas. J Vasc Interv Radiol 2014;25:307-314

23. Chung S, Kim KE, Park MS, Bhagavatula S, Babb J, Axel L. Liver stiffness assessment with tagged MRI of cardiac-induced liver motion in cirrhosis patients. J Magn Reson Imaging 2014;39:1301-1307

24. Brüggmann D, Tchartchian $G$, Wallwiener M, Münstedt $K$, Tinneberg HR, Hackethal A. Intra-abdominal adhesions: definition, origin, significance in surgical practice, and treatment options. Dtsch Arztebl Int 2010;107:769-775 